\title{
Transition of serum cytokines following pancreaticoduodenectomy: A subsidiary study of JAPAN-PD
}

\author{
HIROMICHI MAEDA $^{1 *}$, KEN-ICHI OKADA $^{2 *}$, TSUTOMU FUJII $^{3}$, MARI S. OBA ${ }^{4}$, \\ MANABU KAWAI $^{2}$, SEIKO HIRONO ${ }^{2}$, YASUHIRO KODERA ${ }^{3}$, MASAYUKI SHO ${ }^{5}$, TAKAHIRO AKAHORI ${ }^{5}$, \\ YASUHIRO SHIMIZU ${ }^{6}$, YOSHIYASU AMBO ${ }^{7}$, NARU KONDO $^{8}$, YOSHIAKI MURAKAMI $^{8}$, JIRO OHUCHIDA $^{9}$, \\ HIDETOSHI EGUCHI $^{10}$, HIROAKI NAGANO ${ }^{10}$, JUNICHI SAKAMOTO ${ }^{11}$ and HIROKI YAMAUE ${ }^{2}$ \\ ${ }^{1}$ Cancer Treatment Center, Kochi Medical School, Nankoku, Kochi 783-8505; \\ ${ }^{2}$ Second Department of Surgery, Wakayama Medical University, Wakayama, Wakayama 641-8510; \\ ${ }^{3}$ Department of Gastroenterological Surgery (Surgery II), Nagoya University Graduate School of Medicine, \\ Nagoya, Aichi 466-8560; ${ }^{4}$ Department of Medical Statistics Faculty of Medicine, Toho University, Tokyo 143-8540; \\ ${ }^{5}$ Department of Surgery, Nara Medical University, Kashihara, Nara 634-8521; ${ }^{6}$ Department of Gastroenterological Surgery, \\ Aichi Cancer Center Hospital, Nagoya, Aichi 464-8681; ${ }^{7}$ Department of Surgery, Teine-Keijinkai Hospital, Sapporo, \\ Hokkaido 006-8555; ${ }^{8}$ Department of Surgery, Institute of Biomedical and Health Sciences, Hiroshima University, \\ Hiroshima, Hiroshima 734-8553; ${ }^{9}$ Department of Surgery, Miyazaki Prefectural Miyazaki Hospital, Miyazaki, \\ Miyazaki 880-8510; ${ }^{10}$ Department of Surgery, Graduate School of Medicine, Osaka University, Suita, Osaka 565-0871; \\ ${ }^{11}$ Tokai Central Hospital, Kakamigahara, Gifu 504-8601, Japan
}

Received May 13, 2018; Accepted August 22, 2018

DOI: $10.3892 / 01.2018 .9422$

\begin{abstract}
Our previous study aimed to examine the effect of TJ-100, a widely used herbal medicine, on intestinal function following pancreaticoduodenectomy (PD) in a multicenter, randomized, double-blinded, placebo-controlled manner (JAPAN-PD study). This concomitant study investigated the effect of TJ-100 on serum cytokine levels in patients who underwent PD. Due to the fact that several clinical variables can affect the absolute values of baseline serum cytokine levels, the ratios of the cytokine levels on postoperative day (POD) 3 to those on POD1 were also used for analysis. The present study enrolled 180/224 randomized patients, of whom 91 received TJ-100 and 89 received placebo. As the main findings of the analysis, Wilcoxon signed-rank test revealed no significant difference in the levels of serum cytokines between the groups; however, patients in the TJ-100 group without severe inflammatory complications exhibited significantly higher ratios of interleukin (IL)-4 ( $=123)$, IL-9 ( $=72)$, IL-10 ( $=97)$, PDGF-BB $(n=143)$
\end{abstract}

Correspondence to: Dr Hiroki Yamaue, Second Department of Surgery, Wakayama Medical University, 811-1 Kimiidera, Wakayama, Wakayama 641-8510, Japan

E-mail: yamaue-h@wakayama-med.ac.jp

${ }^{*}$ Contributed equally

Key words: tumor necrosis factor, Kampo, Daikenchuto, herbal medicine, pancreatic cancer and tumor necrosis factor- $\alpha(n=135)$, compared with patients in the Placebo Group $(\mathrm{P}<0.05)$. According to the results of the present study, TJ-100 has an effect on the change in serum cytokine levels from POD1 to POD3 following PD. However, the role of different transition pattern of cytokines in postoperative recovery following PD has to be investigated by further mechanical studies focusing on these extracted cytokines (ClinicalTrials.gov; no. NCT01607307; May 30, 2012).

\section{Introduction}

TJ-100 (Daikenchuto, or TU-100), which comprises three different medicinal herbs, is widely used in clinical practice to treat 'abdominal bloating and cold sensation in the abdomen' $(1,2)$. Clinical studies support the use of TJ-100 in the perioperative period to accelerate the recovery of gastrointestinal function (3-6). The beneficial effect of TJ-100 is mediated by enteric/sensory nerve stimulation via acetylcholine receptors and/or serotonin receptors $(7,8)$ and by the release of adrenomedullin from epithelial cells (9-11). Moreover, TJ-100 decrease the level of postoperative inflammatory cytokines, ameliorating inflammation and postoperative ileus in animal model (12).

JAPAN-PD study, a multicenter, randomized, double-blinded, and placebo-control trial involving nearly 300 patients, was conducted to study the effect of TJ-100 on recovery of gastrointestinal function after pancreaticoduodenectomy (PD) (13). In contrast to the hypothesis, perioperative administration of TJ-100 provided no positive effect on time to first postoperative flatus and occurrence rate of paralytic ileus. Concurrently, the cytokine levels in 180 of the trial patient group were investigated, measuring the levels in serum and drainage 
fluid on postoperative day (POD)1 and POD3. Consistently, we found the lack of significant difference in 27 cytokine levels for both serum and drainage fluid between the TJ-100- and placebo-treated patients $((\mathrm{n}=180, \mathrm{P}>0.05))(13)$.

However, we noted that the cytokine levels differed substantially among individuals. The range of factors potentially influencing cytokine levels, including gene polymorphisms $(14,15)$, malignancy itself (16), and different degree of pre/postoperative inflammation, might have masked the effect of TJ-100. We also considered that cytokine levels of drainage fluid are not suitable for evaluating the effects of TJ-100, because they would be more influenced by the local environment near the tip of the tube than by the whole environment or systemic response. Thus, we determined to re-analyze the effect of TJ-100 using the data from the previous study, but with a special focus on the serum levels and efforts to minimize the influence of other potentially confounding factors.

\section{Materials and methods}

Trial design and patient recruitment. The details of the protocol and patient recruitment were described previously (13). Patients aged 20 years or older, who were undergoing PD for periampullary tumor or pancreas head tumors, were recruited. The patients were randomized according to four clinical variables: i) type of primary tumor; ii) presence of neoadjuvant chemotherapy; iii) preservation of gastric pylorus; and iv) presence of enteral alimentation via a feeding tube. Administration of TJ-100 ( $15 \mathrm{~g}$ /day divided into three doses; before every meal or every 8 h) or placebo was initiated three days before surgery, and continued until POD14. Patient serum was collected on POD1 and POD3, and assayed for 27 different cytokines by LSI Medience Corporation (Tokyo, Japan) using the Human Cytokine 27-plex assay (Bio-Rad Laboratories, Inc., Hercules, CA, USA) and Bio-Plex 200 System (Bio-Rad Laboratories, Inc.).

The study protocol was registered at UMIN Clinical Trial Registry (no. 000007975) and at ClinicalTrials.gov (no. NCT01607307). The institutional review board of each participating institute approved the trial protocol, and the randomization and intervention was started after obtaining the patients' written informed consent. Additional written informed consent was obtained for the present study.

Statistical analysis. The characteristic features were compared between the patients receiving TJ-100 (TJ-100 Group) and placebo (Placebo Group) using Student's t-test or Fisher's exact tests. First, we compared the levels of serum cytokines on POD1 and POD3 between the groups by using the Wilcoxon signed-rank test. Second, we compared ratios of the cytokine levels on POD3 to those on POD1 for further analysis using Wilcoxon signed-rank test. Note that the values under the measurement threshold were excluded from the analysis, because the assignment of lower limit may significantly distort the ratios. P-values of $<0.05$ was considered significant. The statistical analysis was performed using SAS 9.4 (SAS Institute, Inc., Cary, NC, USA).

\section{Results}

Clinical variables. We analyzed clinical data of the patients who participated in this additional explorative study after dividing them into the TJ-100 Group and Placebo Group (Fig. 1 and Table I). As in the original JAPAN-PD study (13), the TJ-100 Group included more patients of an advanced age than did the Placebo Group (69.4 \pm 8.0 years vs. $65.1 \pm 11.4$, $\mathrm{P}=0.039$ ). We consider this difference was coincidentally formed because the age was not used as a balancing variable of randomization. Other clinical factors that could potentially alter the cytokine levels did not differ between the groups.

Absolute values of serum cytokines. The values under the measurement threshold were excluded in this analysis. As a result, IL-2 and IL-15 were analyzed for only a small number of the patients, and thus statistical comparison was invalid. As previously reported (13), Wilcoxon signed-rank test revealed no significant difference in cytokine levels between the groups for POD1 (Table II) or POD3.

Of note, the cytokine levels ranged widely among individuals. In TNF- $\alpha$ on POD1, for instance, $1,072 \mathrm{pg} / \mathrm{ml}$ was observed as a maximum value, while the lower level was below the lower measurement limit (20.64 pg/ml). Similarly, the ranges of other cytokines levels were found quite large after PD.

\section{Changes in serum cytokine levels after treatment}

Whole groups. The change in serum cytokine level was defined by the ratio of cytokine levels on POD3 divided by those on POD1 in each individual, and then the ratios were compared between the groups. The ratios over time of IL-4, IL-9, and IL-17 were higher in the TJ-100 Group compared to the Placebo Group, suggesting that these cytokines increased more from POD1 to POD3 following treatment with TJ-100 than with placebo.

Patients without Grade B/C pancreatic fistula or abdominal abscess. The same analysis was performed for the patients without complications related to strong abdominal inflammation, namely Grade B/C pancreatic fistula or abdominal abscess. In this subgroup, the cytokine level ratios were significantly higher for IL-4, IL-9, IL-10, PDGF-BB, and TNF- $\alpha$ in the TJ-100 Group than the Placebo Group (Table III). For IL-4, the median ratio was 1.7 in TJ-100 Group, compared to 1.1 with placebo, suggesting that serum level of IL-4 more increased from POD1 to POD3 following TJ-100 treatment (Fig. 2A). For IL-9, IL-10, and PDGF-BB, the median ratios slightly exceeded 1.0 in the TJ-100 Group, while the median ratios of these cytokines were below 1.0 in the Placebo Group (Fig. 2B-D). For TNF- $\alpha$, a statistical difference was demonstrated, although the difference in median ratio was quite small between the groups (TJ-100 Group vs. Placebo Group: 1.2 vs. $1.0, \mathrm{P}=0.041$; Fig. $2 \mathrm{E})$.

Patients with Grade B/C pancreatic fistula or abdominal abscess. There was no significant difference in transition of the serum cytokine level when patients with Grade B/C pancreatic fistula or abdominal abscess were analyzed.

The effect of patient age on ratios of cytokine levels. We also assessed the impact of patient age on the cytokine levels and the ratios. Patients were divided into two groups, younger than 70 or 70 years and older. Comparison of the ratios for IL-4, IL-9, IL-10, PDGF-BB, and TNF- $\alpha$ showed no significant difference 
Table I. Comparison between clinical variables and the TJ-100 and Placebo Groups.

\begin{tabular}{lcc}
\hline Patient characteristic & TJ-100, $=91$ & Placebo, n=89 \\
\hline Age, mean \pm SD (years) & $69.4 \pm 8.0$ & $65.1 \pm 11.4$ \\
Sex, male/female (\%) & $50 / 41(55 / 45)$ & $59 / 30(66 / 34)$ \\
BMI, mean \pm SD $\left(\mathrm{kg} / \mathrm{m}^{2}\right)$ & $22.1 \pm 3.0$ & $22.3 \pm 3.5$ \\
Hx of abdominal surgery (\%) & $41(45)$ & $30(34)$ \\
Tumor characteristics & & 0.130 \\
Pancreatic Ca, yes $(\%)$ & $52(57)$ & $51(57)$ \\
Preoperative treatment, yes (\%) & $74(81)$ & $71(80)$ \\
Tumor stage, /I/II/III/Iva/IVb/ND (\%) & $3 / 12 / 7 / 25 / 27 / 3 / 13$ & $3 / 9 / 6 / 25 / 24 / 4 / 18$ \\
& $(3 / 13 / 8 / 28 / 30 / 3 / 14)$ & $(3 / 10 / 7 / 28 / 27 / 20)$ \\
Surgical variable & & 0.130 \\
Surgical time, mean \pm SD (min) & $416.1 \pm 102.5$ & 0.303 \\
Bleeding, mean \pm SD (ml) & $719.1 \pm 617.9$ & $405.2 \pm 112.6$ \\
Use of epidural anesthesia (\%) & $61(67)$ & $696.1 \pm 694.6$ \\
Lymph node dissection, D0/D1/D2/D3 (\%) & $1 / 12 / 78 / 0$ & $65(73)$ \\
Dissection of nerve plexus around the SMA (\%) & $(1 / 13 / 86 / 0)$ & $2 / 8 / 78 / 1$ \\
\hline
\end{tabular}

SD, standard deviation; BMI, body mass index; SMA, superior mesenteric plexus.

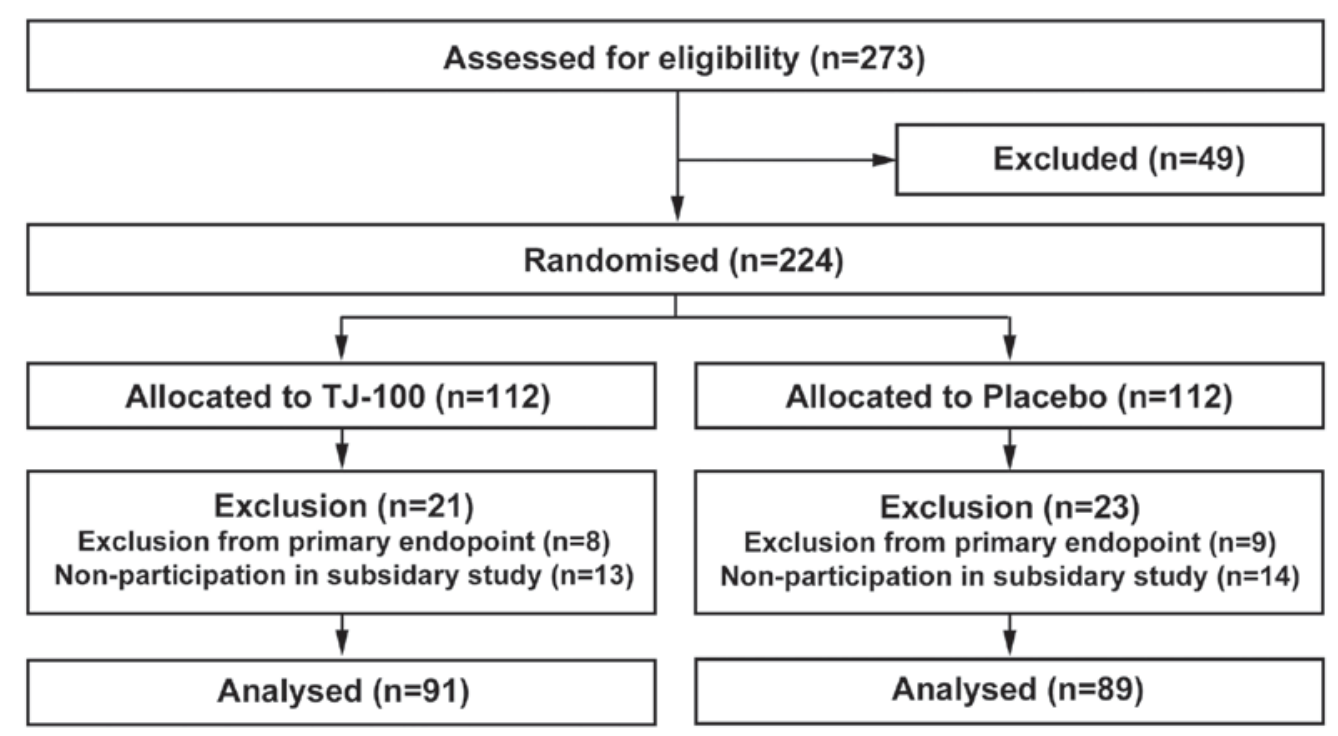

Figure 1. Flow diagram of the study. Among 273 patients, 49 were excluded from randomization due to exclusion criteria ( $\mathrm{n}=41)$, ineligibility (n=4) and declinature $(n=4)$. Following randomization, a total of 17 patients were excluded from the primary analysis, as 13 patients did not undergo pancreatoduodenectomy, three did not receive protocol treatment and one patient withdrew consent.

between younger and elder patients (Wilcoxon signed-rank test, $\mathrm{P}=0.75,0.465,0.695,0.297$, and 0.688 , respectively).

\section{Discussion}

There are two intriguing findings in the initial analysis of this study. First, comparing the serum cytokine levels by Wilcoxon signed-rank testing revealed no significant difference at POD1 or POD3 between the patients receiving TJ-100 treatment and those receiving placebo. Second, the cytokine levels significantly varied among the individuals even though the patients underwent similar, invasive surgical procedures. The increase in cytokine levels following surgery was quite small in some patients, even with severe complications, while other patients had substantially increased levels of the cytokines despite an uneventful postoperative recovery (data not shown).

Preoperative tumor burden and inflammation might explain these observations in part, because malignancies have been shown to alter the levels of cytokines compared to benign diseases needing surgical interventions (16). Namely, 
Table II. Comparison of serum cytokine levels on POD1 between the TJ-100 and Placebo Groups.

\begin{tabular}{|c|c|c|c|c|c|c|c|}
\hline \multirow[b]{2}{*}{ Factor } & \multicolumn{3}{|c|}{ TJ-100 (n=91) } & \multicolumn{3}{|c|}{ Placebo $(\mathrm{n}=89)$} & \multirow[b]{2}{*}{ P-value } \\
\hline & $\mathrm{n}$ & Median & (Min-Max) & $\mathrm{n}$ & Median & (Min-Max) & \\
\hline \multicolumn{8}{|l|}{ Interleukin } \\
\hline IL-2 & 5 & 45.5 & $(35.7-739.2)$ & 13 & 37.2 & (17.9-1250.9) & 0.200 \\
\hline IL-7 & 90 & 14.7 & $(2.6-129.7)$ & 89 & 18 & $(3.1-248.3)$ & 0.316 \\
\hline IL-9 & 54 & 22.6 & (10.3-4061.4) & 52 & 25.4 & $(11-2143.1)$ & 0.183 \\
\hline IL-13 & 91 & 7.5 & $(1-124.4)$ & 88 & 7.6 & $(1.6-152)$ & 0.466 \\
\hline IL-15 & 2 & 99.3 & $(34.1-164.5)$ & 4 & 166.8 & $(74.6-595.7)$ & 0.487 \\
\hline IL-5 & 91 & 5.9 & $(0.5-108.1)$ & 89 & 6.6 & $(0.5-230.1)$ & 0.331 \\
\hline GM-CSF & 52 & 34.1 & $(13.8-146.8)$ & 45 & 51.5 & (14.4-173.9) & 0.065 \\
\hline IL-1 $\beta$ & 78 & 5.7 & $(2.2-53.3)$ & 77 & 6.5 & $(2.3-51.2)$ & 0.284 \\
\hline IL-6 & 91 & 216.1 & $(66.4-2024.7)$ & 89 & 208.4 & $(20.2-3084.5)$ & 0.773 \\
\hline IL-12 & 67 & 38.6 & $(11.2-635.8)$ & 63 & 44.8 & $(11.2-747.2)$ & 0.278 \\
\hline IL-17 & 56 & 102.8 & (34.3-1384.4) & 54 & 169.9 & $(33-602.9)$ & 0.354 \\
\hline IL-1Ra & 87 & 339.2 & $(36.4-2706.8)$ & 85 & 319.9 & $(56.5-11374.9)$ & 0.547 \\
\hline IL-4 & 82 & 4.1 & $(1.2-46.7)$ & 77 & 4.9 & $(1.2-20)$ & 0.152 \\
\hline IL-10 & 80 & 34.5 & $(8.7-271.4)$ & 74 & 41.2 & $(8.7-1152.8)$ & 0.121 \\
\hline \multicolumn{8}{|l|}{ Chemokine } \\
\hline IL-8 & 91 & 55.2 & $(22.4-422.7)$ & 89 & 56.5 & $(20-188.1)$ & 0.925 \\
\hline IP-10 & 91 & 327.2 & $(69.3-2574.7)$ & 89 & 306 & $(54.3-2452.9)$ & 0.857 \\
\hline MCP-1 & 91 & 91.9 & $(28.7-453.5)$ & 89 & 96 & $(34.8-1877.4)$ & 0.854 \\
\hline MIP- $1 \alpha$ & 90 & 8.6 & $(2.5-34.9)$ & 89 & 8.7 & $(2-31.3)$ & 0.640 \\
\hline MIP-1 $\beta$ & 91 & 114.8 & $(36.7-302)$ & 89 & 100.5 & $(27-287.4)$ & 0.381 \\
\hline RANTES & 91 & $9,121.7$ & $(740.1-154119.1)$ & 89 & $8,984.5$ & $(1263.2-212597.8)$ & 0.907 \\
\hline Eotaxin & 44 & 56.6 & $(32.1-257.8)$ & 47 & 56.5 & $(28.2-1500.3)$ & 0.821 \\
\hline \multicolumn{8}{|c|}{ Growth factor } \\
\hline Basic FGF & 72 & 61.4 & $(13.6-379.4)$ & 70 & 64.4 & $(14.4-289.7)$ & 0.202 \\
\hline PDGF-BB & 91 & 444.8 & $(9.1-5535.9)$ & 88 & 435.6 & $(31.3-11803.5)$ & 0.582 \\
\hline VEGF & 71 & 54.7 & $(13.3-648.1)$ & 64 & 69.5 & $(12.5-359.8)$ & 0.165 \\
\hline \multicolumn{8}{|c|}{ Hematopoietic factor } \\
\hline G-CSF & 88 & 123.5 & $(36.6-1240.3)$ & 87 & 122.9 & $(33.7-911.1)$ & 0.946 \\
\hline Interferon- $\gamma$ & 91 & 114.2 & $(21.2-1451.8)$ & 88 & 137.5 & $(21.2-854.5)$ & 0.324 \\
\hline TNF $\alpha$ & 90 & 91.2 & $(22-1072.7)$ & 84 & 101.7 & $(25.6-784.6)$ & 0.370 \\
\hline
\end{tabular}

POD, postoperative day; IL, interleukin; IP, interferon $\gamma$-induced protein 10; MCP, monocyte chemoattractant protein 1; MIP, macrophage inflammatory protein; RANTES, regulated on activation, normal T cell expressed and secreted; FGF, fibroblast growth factor; PDGF, platelet-derived growth factor; VEGF, vascular endothelial growth factor; G-CSF, granulocyte colony-stimulating factor; TNF, tumor necrosis factor.

the preoperative levels of IL-1 $\beta$, IL-7, IL-8, G-CSF, IFN- $\gamma$, and TNF- $\alpha$ were significantly increased in patients with colon cancer, while these cytokines remained low in patients with other diseases (16). The altered cytokine levels before surgery were likely to have occurred in the present study due to different states of cancer advancement and/or different types of tumors, and such preoperative differences might have consequently hampered detection of the effects of TJ-100. Another possible explanation for the wide range of cytokine levels observed in the present study is polymorphisms of genes encoding cytokines. For instance, production of IL-1 $\beta$, TNF- $\alpha$, and IL-10 from peripheral blood mononuclear cells (PBMCs) obtained from pancreatic cancer patients or healthy volunteers was evidently affected by particular gene polymorphisms $(14,15)$.
Furthermore, the relationship between gene polymorphisms of proinflammatory cytokines and their serum levels and short-term operative results have been demonstrated in the field of esophagectomy (17). Unfortunately, these two potential differences in clinical background were not addressed in the present study. At least, our findings clearly suggested that application of serum cytokines as markers for the strength of inflammation or severity of complication after PD might not be as straightforward as previously considered.

By addressing the aforementioned potential confounding factors by comparing ratios of cytokine levels on POD3 to those on POD1, we found that changes in IL-10, IL-4, IL-9, TNF- $\alpha$, and PDGF-BB were significantly reduced by TJ-100 in patients without severe complications. In animal models, 
Table III. Change in serum cytokine levels between POD1 and POD3 in patients without severe abdominal inflammation.

\begin{tabular}{|c|c|c|c|c|c|c|c|}
\hline \multirow[b]{3}{*}{ Factor } & \multicolumn{3}{|c|}{ TJ-100 (n=74) } & \multicolumn{3}{|c|}{ Placebo $(n=70)$} & \multirow[b]{3}{*}{ P-value } \\
\hline & \multicolumn{3}{|c|}{ (Value on POD1)/(Value on POD3) } & \multicolumn{3}{|c|}{ (Value on POD1)/(Value on POD3) } & \\
\hline & $\mathrm{n}$ & Median & (Min-Max) & $\mathrm{n}$ & Median & (Min-Max) & \\
\hline \multicolumn{8}{|l|}{ Interleukin } \\
\hline IL-2 & 5 & 1.1 & $(0.5-1.4)$ & 4 & 0.7 & $(0.4-1)$ & 0.178 \\
\hline IL-7 & 73 & 1.3 & $(0.3-16.5)$ & 70 & 1.2 & $(0.3-7.2)$ & 0.164 \\
\hline IL-9 & 37 & 1.2 & $(0.4-6.2)$ & 35 & 0.8 & $(0.2-3.5)$ & 0.031 \\
\hline IL-13 & 74 & 1.9 & $(0.4-18.1)$ & 69 & 1.5 & $(0.2-14.3)$ & 0.177 \\
\hline IL-15 & 0 & - & $(0.4-18.1)$ & 2 & 0.9 & $(0.9-0.9)$ & - \\
\hline IL-5 & 74 & 1.5 & $(0.2-7.3)$ & 70 & 1.1 & $(0.2-5.4)$ & 0.095 \\
\hline GM-CSF & 26 & 0.8 & $(0.1-4.5)$ & 26 & 0.6 & $(0.2-2.8)$ & 0.185 \\
\hline IL-1 $\beta$ & 60 & 1.2 & $(0.3-7.4)$ & 59 & 1 & $(0.3-4.9)$ & 0.140 \\
\hline IL-6 & 73 & 0.3 & $(0-50.5)$ & 70 & 0.3 & $(0-1)$ & 0.785 \\
\hline IL-12 & 47 & 1.2 & $(0.3-3.8)$ & 42 & 0.8 & $(0.2-4.3)$ & 0.105 \\
\hline IL-17 & 39 & 1.2 & $(0.2-5.2)$ & 34 & 0.8 & $(0.1-7)$ & 0.094 \\
\hline IL-1Ra & 66 & 1.1 & $(0.3-10.3)$ & 66 & 1 & $(0.2-7.8)$ & 0.116 \\
\hline IL-4 & 63 & 1.7 & $(0.5-7.9)$ & 60 & 1.1 & $(0.2-7.7)$ & 0.015 \\
\hline IL-10 & 48 & 1.1 & $(0.2-5.4)$ & 49 & 0.8 & $(0.2-6.6)$ & 0.032 \\
\hline \multicolumn{8}{|l|}{ Chemokine } \\
\hline IL-8 & 74 & 0.8 & $(0.1-14.1)$ & 70 & 0.8 & $(0.3-4.7)$ & 0.444 \\
\hline IP-10 & 74 & 1.1 & $(0.4-27)$ & 70 & 1.1 & $(0.4-12.2)$ & 0.516 \\
\hline MCP-1 & 74 & 0.7 & $(0.3-30.1)$ & 70 & 0.7 & $(0.2-2.7)$ & 0.461 \\
\hline MIP- $1 \alpha$ & 73 & 1.1 & $(0.5-6.5)$ & 70 & 1.1 & $(0.3-6.5)$ & 0.230 \\
\hline MIP-1 $\beta$ & 74 & 0.9 & $(0.5-3.8)$ & 70 & 0.8 & $(0.3-2.4)$ & 0.430 \\
\hline RANTES & 74 & 0.9 & $(0.2-8.8)$ & 70 & 0.8 & $(0-18.2)$ & 0.345 \\
\hline Eotaxin & 23 & 0.8 & $(0.4-2.1)$ & 27 & 0.9 & $(0.2-1.9)$ & 0.668 \\
\hline \multicolumn{8}{|l|}{ Growth factors } \\
\hline Basic FGF & 53 & 1.1 & $(0.3-12)$ & 48 & 0.8 & $(0.1-5.9)$ & 0.195 \\
\hline PDGF-BB & 74 & 1.2 & $(0.1-22.8)$ & 69 & 0.8 & $(0-16.3)$ & 0.049 \\
\hline VEGF & 50 & 1.2 & $(0.3-10.6)$ & 42 & 1 & $(0.1-5.1)$ & 0.195 \\
\hline \multicolumn{8}{|c|}{ Hematopoietic factor } \\
\hline G-CSF & 69 & 1.1 & $(0.1-93.5)$ & 66 & 1 & $(0.2-7.5)$ & 0.370 \\
\hline Interferon- $\gamma$ & 74 & 1.2 & $(0.2-8)$ & 69 & 1.1 & $(0.3-6.3)$ & 0.276 \\
\hline TNF- $\alpha$ & 70 & 1.2 & $(0.3-8.6)$ & 65 & 1 & $(0.2-4.2)$ & 0.041 \\
\hline
\end{tabular}

POD, postoperative day; IL, interleukin; IP, interferon $\gamma$-induced protein 10; MCP, monocyte chemoattractant protein 1; MIP, macrophage inflammatory protein; RANTES, regulated on activation, normal T cell expressed and secreted; FGF, fibroblast growth factor; PDGF, platelet-derived growth factor; VEGF, vascular endothelial growth factor; G-CSF, granulocyte colony-stimulating factor; TNF, tumor necrosis factor.

TJ-100 decreased expression of TNF- $\alpha$ in target organs $(12,18)$, reducing postoperative paralytic ileus and bacterial translocation. Furthermore, TJ-100 decreased levels of serum C-reactive protein in patients following surgery for colon/rectal disease (19), suggesting that TJ-100 could ameliorate inflammatory responses. In contrast, the present analysis demonstrated a higher increase in TNF- $\alpha$ level from POD3 to POD1 in patients who received TJ-100 compared to the Placebo Group, indicating that TJ-100 might enhance the early inflammatory response after PD. The contradiction between previous observations and ours may be derived from the different extents of surgical stress. While the uncontrolled expression of TNF- $\alpha$ is associated with an overwhelming inflammatory response, multiple-organ dysfunction, and impaired tissue recovery, extensive suppression of TNF- $\alpha$ results in frequent occurrence of infectious complications after surgery (20). The appropriate inflammatory response elicited against surgical stress is clearly essential for immunological defense and tissue repair, and the biological influence of sustained serum levels of TNF- $\alpha$ in patients receiving PD and TJ-100 should be investigated further.

IL-4, IL-10, and PDGF-BB are associated with wound healing (21-24), while increased IL-9 might enhance intestinal muscle contractility and protect against bacterial shock by modulating inflammatory and anti-inflammatory 

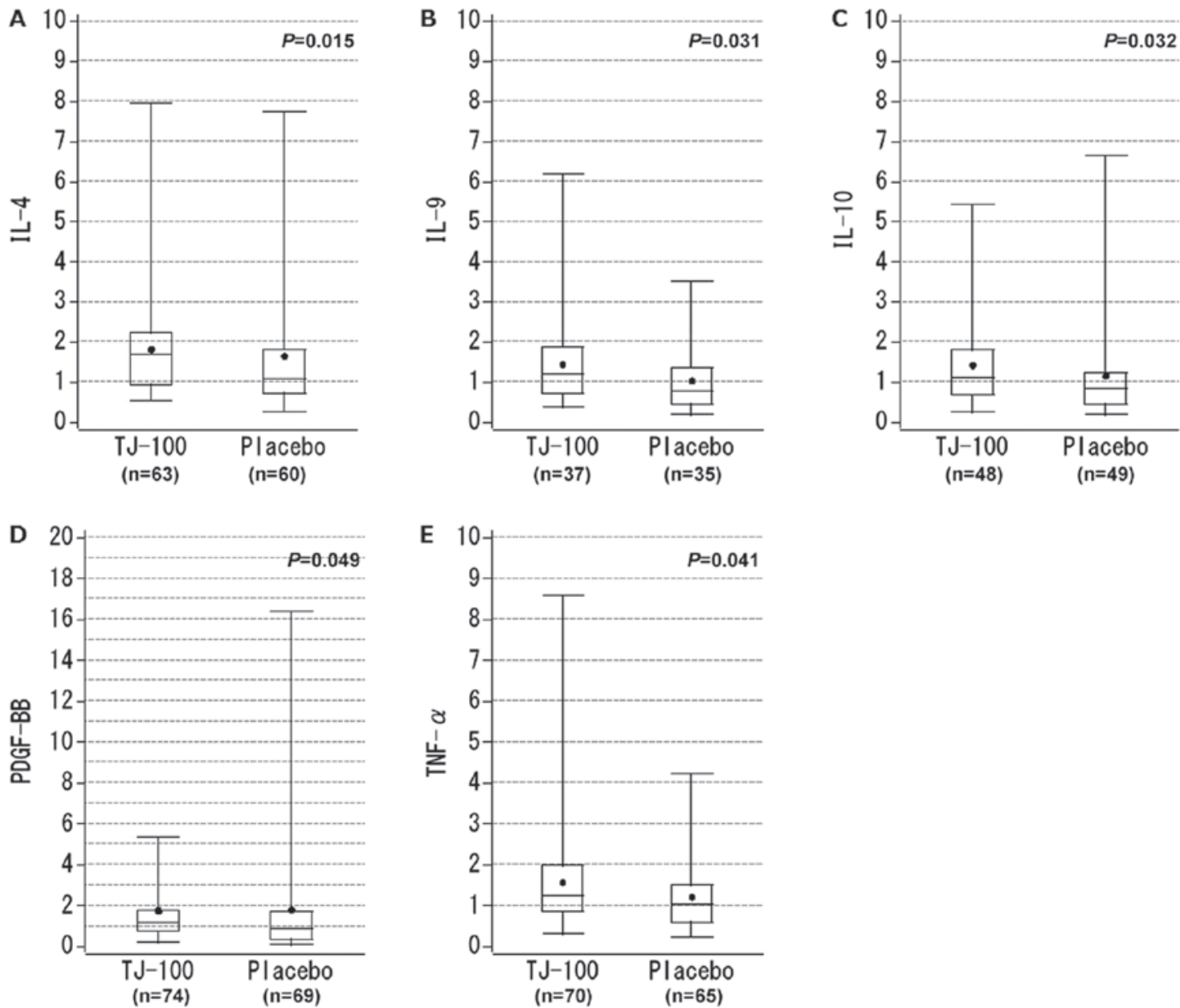

Figure 2. The ratios of cytokine levels on POD3 to those of POD1 were compared between the TJ-100 Group and the Placebo Group. A significant difference was observed in (A) IL-4, (B) IL-9, (C) IL-10, (D) PDGF-BB and (E) TNF- $\alpha$. Dots represent the means and bars inside the boxes represent the medians of the ratios. IL, interleukin; TNF, tumor necrosis factor.

mediators $(25,26)$. These observations are partially consistent with our previous clinical study of 45 patients undergoing PD (27); however, the overall clinical significance of different changes in cytokine levels due to TJ-100 administration was not determined by the present study.

Several limitations related to this analysis should be noted in addition to the lack of baseline (preoperative) cytokine levels and information of gene polymorphisms. First, the levels of cytokines were under the detection threshold in several patients, precluding these samples from the final analysis. Similarly, the number of patients with severe complications was not large enough to detect statistical differences of cytokine transitions in the present study. Finally, the level of the cytokines at the later phase of recovery, such as 7 days after operation, could have brought better understanding of the effect of TJ-100.

In conclusion, we examined the levels of 27 cytokines after PD and their transition from POD1 to POD3 in a large number of the patients enrolled in a multi-institutional, prospective trial. At initial assessment, a wide range of cytokine levels among individuals after PD was found, suggesting the difficulties of using the cytokines levels to assume the extent of surgical stress. However, oral administration of TJ-100 altered the postoperative changes in serum levels of IL-4, IL-9, IL-10, TNF- $\alpha$, and PDGF-BB. With consideration of gene polymorphism and preoperative inflammatory status, studies using
TJ-100 under surgical stress should be performed to elucidate the role of different transition pattern of cytokines.

\section{Acknowledgements}

Not applicable.

\section{Funding}

The present study was supported by the non-profit organization Epidemiological and Clinical Research Information Network (ECRIN).

\section{Availability of data and materials}

All data generated or analyzed during this study are included in this published article.

\section{Authors' contributions}

All authors read and approved the final manuscript. HM performed data analysis, data interpretation, figure and table creation, and wrote the manuscript. KO participated in study design, study protocol creation, literature search, data collection, data analysis and data interpretation. TF participated in 
study design, data collection and manuscript revision. MSO participated in study protocol creation, data analysis and figure creation. MK, SH, YK, MS, TA, YS, YA, NK, YM, JO, HE and HN participated in study design, study protocol creation, data collection and manuscript revision. JS and HY participated in study design, study protocol creation, literature search, data collection, data analysis, data interpretation, figure and table creation, and manuscript writing.

\section{Ethics approval and consent to participate}

All patients provided written informed consent prior to study inclusion. The institutional review board of each participating institute approved the trial protocol.

\section{Patient consent for publication}

All patients provided written informed consent.

\section{Competing interests}

The authors declare that they have no competing interests.

\section{References}

1. Kono T, Shimada M, Yamamoto M, Kaneko A, Oomiya Y, Kubota K, Kase Y, Lee K and Uezono Y: Complementary and synergistic therapeutic effects of compounds found in Kampo medicine: Analysis of daikenchuto. Front Pharmacol 6: 159, 2015.

2. Manabe N, Camilleri M, Rao A, Wong BS, Burton D, Busciglio I, Zinsmeister AR and Haruma K: Effect of daikenchuto (TU-100) on gastrointestinal and colonic transit in humans. Am J Physiol Gastrointest Liver Physiol 298: G970-G975, 2010.

3. Yoshikawa K, Shimada M, Wakabayashi G, Ishida K, Kaiho T, Kitagawa Y, Sakamoto J, Shiraishi N, Koeda K, Mochiki E, et al: Effect of daikenchuto, a traditional japanese herbal medicine, after total gastrectomy for gastric cancer: A multicenter, randomized, double-blind, placebo-controlled, phase II trial. J Am Coll Surg 221: 571-578, 2015.

4. Shimada M, Morine Y, Nagano H, Hatano E, Kaiho T, Miyazaki M, Kono T, Kamiyama T, Morita S, Sakamoto J, et al: Effect of TU-100, a traditional Japanese medicine, administered after hepatic resection in patients with liver cancer: A multi-center, phase III trial (JFMC40-1001). Int J Clin Oncol 20 95-104, 2015 .

5. Katsuno H, Maeda K, Kaiho T, Kunieda K, Funahashi K, Sakamoto J, Kono T, Hasegawa H, Furukawa Y, Imazu Y, et al Clinical efficacy of Daikenchuto for gastrointestinal dysfunction following colon surgery: A randomized, double-blind, multicenter, placebo-controlled study (JFMC39-0902). Jpn J Clin Oncol 45: 650-656, 2015.

6. Katsuno H, Maeda K, Ohya M, Yoshioka K, Tsunoda A, Koda K, Matsuoka H, Ohge H, Morita S, Saji S, et al: Clinical pharmacology of daikenchuto assessed by transit analysis using radiopaque markers in patients with colon cancer undergoing open surgery: A multicenter double-blind randomized placebo-controlled study (JFMC39-0902 additional study). J Gastroenterol 51: 222-229, 2016.

7. Satoh K, Hayakawa T, Kase Y, Ishige A, Sasaki H, Nishikawa S, Kurosawa S, Yakabi K and Nakamura T: Mechanisms for contractile effect of Dai-kenchu-to in isolated guinea pig ileum. Dig Dis Sci 46: 250-256, 2001.

8. Fukuda H, Chen C, Mantyh C, Ludwig K, Pappas TN and Takahashi T: The herbal medicine, Dai-Kenchu-to, accelerates delayed gastrointestinal transit after the operation in rats. J Surg Res 131: 290-295, 2006.

9. Kono T, Kaneko A, Omiya Y, Ohbuchi K, Ohno N and Yamamoto M: Epithelial transient receptor potential ankyrin 1 (TRPA1)-dependent adrenomedullin upregulates blood flow in rat small intestine. Am J Physiol Gastrointest Liver Physiol 304: G428-G436, 2013
10. Tsuchiya K, Kubota K, Ohbuchi K, Kaneko A, Ohno N, Mase A, Matsushima H, Yamamoto M, Miyano K, Uezono Y and Kono T: Transient receptor potential ankyrin 1 agonists improve intestinal transit in a murine model of postoperative ileus. Neurogastroenterol Motil 28: 1792-1805, 2016.

11. Kono T, Kaneko A, Hira Y, Suzuki T, Chisato N, Ohtake N, Miura $\mathrm{N}$ and Watanabe T: Anti-colitis and -adhesion effects of daikenchuto via endogenous adrenomedullin enhancement in Crohn's disease mouse model. J Crohns Colitis 4: 161-170, 2010.

12. Endo M, Hori M, Ozaki H, Oikawa T and Hanawa T: Daikenchuto, a traditional Japanese herbal medicine, ameliorates postoperative ileus by anti-inflammatory action through nicotinic acetylcholine receptors. J Gastroenterol 49: 1026-1039, 2014.

13. Okada K, Kawai M, Hirono S, Fujii T, Kodera Y, Sho M, Nakajima Y, Satoi S, Kwon AH, Shimizu Y, et al: Evaluation of the efficacy of daikenchuto (TJ-100) for the prevention of paralytic ileus after pancreaticoduodenectomy: A multicenter, double-blind, randomized, placebo-controlled trial. Surgery 159: 1333-1341, 2016.

14. Barber MD, Powell JJ, Lynch SF, Fearon KC and Ross JA: A polymorphism of the interleukin-1 beta gene influences survival in pancreatic cancer. Br J Cancer 83: 1443-1447, 2000.

15. Warlé MC, Farhan A, Metselaar HJ, Hop WC, Perrey C, Zondervan PE, Kap M, de Rave S, Kwekkeboom J, Ijzermans JN, et al: Cytokine gene polymorphisms and acute human liver graft rejection. Liver Transpl 8: 603-611, 2002.

16. Crucitti A, Corbi M, Tomaiuolo PM, Fanali C, Mazzari A, Lucchetti D, Migaldi M and Sgambato A: Laparoscopic surgery for colorectal cancer is not associated with an increase in the circulating levels of several inflammation-related factors. Cancer Biol Ther 16: 671-677, 2015.

17. Sakamoto K, Oka M, Yoshino S, Hazama S, Takeda S, Yoshimura K, Okayama N and Hinoda Y: Relationship between cytokine gene polymorphisms and risk of postoperative pneumonia with esophageal cancer. J Gastrointest Surg 18: 1247-1253, 2014.

18. Yoshikawa K, Kurita N, Higashijima J, Miyatani T, Miyamoto H, Nishioka M and Shimada M: Kampo medicine 'Dai-kenchu-to' prevents bacterial translocation in rats. Dig Dis Sci 53: 1824-1831, 2008.

19. Osawa G, Yoshimatsu K, Yokomizo H, Otani T, Matsumoto A, Nakayama M and Ogawa K: The clinical effects of dai-kenchu-to on postoperative intestinal movement and inflammatory reaction in colorectal surgery. Hepatogastroenterology 62: 807-810, 2015.

20. Yang ZP, Hong L, Wu Q, Wu KC and Fan DM: Preoperative infliximab use and postoperative complications in Crohn's disease: A systematic review and meta-analysis. Int J Surg 12: 224-230, 2014

21. Kieran I, Knock A, Bush J, So K, Metcalfe A, Hobson R, Mason T, O'Kane S and Ferguson M: Interleukin-10 reduces scar formation in both animal and human cutaneous wounds: Results of two preclinical and phase II randomized control studies. Wound Repair Regen 21: 428-436, 2013.

22. Salmon-Ehr V, Ramont L, Godeau G, Birembaut P, Guenounou M, Bernard P and Maquart FX: Implication of interleukin-4 in wound healing. Lab Invest 80: 1337-1343, 2000.

23. Gupta M, Poonawala T, Farooqui M, Ericson ME and Gupta K: Topical fentanyl stimulates healing of ischemic wounds in diabetic rats. J Diabetes 7: 573-583, 2015.

24. Bhansali A, Venkatesh S, Dutta P, Dhillon MS, Das S and Agrawal A: Which is the better option: Recombinant human PDGF-BB $0.01 \%$ gel or standard wound care, in diabetic neuropathic large plantar ulcers off-loaded by a customized contact cast? Diabetes Res Clin Pract 83: e13-e16, 2009.

25. Khan WI, Richard M, Akiho H, Blennerhasset PA, Humphreys NE, Grencis RK, Van Snick J and Collins SM: Modulation of intestinal muscle contraction by interleukin-9 (IL-9) or IL-9 neutralization: Correlation with worm expulsion in murine nematode infections. Infect Immun 71: 2430-2438, 2003.

26. Grohmann U, Van Snick J, Campanile F, Silla S, Giampietri A, Vacca C, Renauld JC, Fioretti MC and Puccetti P: IL-9 protects mice from Gram-negative bacterial shock: Suppression of TNF-alpha, IL-12, and IFN-gamma, and induction of IL-10. J Immunol 164: 4197-4203, 2000.

27. Okada K, Kawai M, Hirono S, Miyazawa M, Shimizu A, Kitahata $\mathrm{Y}$ and Yamaue $\mathrm{H}$ : Perioperative administration of Daikenchuto (TJ-100) reduces the postoperative paralytic ileus in patients with pancreaticoduodenectomy. Hepatogastroenterology 62: 466-471, 2015. 